\title{
Batik Fractal: Marriage of Art and Science
}

\author{
Yun Hariadi ${ }^{1}$, Muhamad Lukman ${ }^{1}$ \& Achmad Haldani Destiarmand ${ }^{2}$ \\ ${ }^{1}$ Pixel People Project, Jl. Jakarta KKP, Kav. 30, Bandung 40217, Indonesia \\ ${ }^{2}$ Craft and Tradition Research Group, Faculty of Visual Art and Design, Bandung \\ Institute of Technology, Jalan Ganesa No.10, Bandung 40132, Indonesia \\ Email: email@yunhariadi.com
}

\begin{abstract}
By applying Fourier Transformation, this paper shows that batik has fractal characteristic. This character is shown in batik's fractal dimension between 1 and 2 . The isen process in batik is one factor that contributes to create self affine as one of fractal's important characteristic. Anova Test for fractal dimension in this method classifies batik according to patterns and its region. Fractal in batik shows the presence of complexity in traditional art. This complexity arises because the effort to obey pakem rule and media. Furthermore, the presence of fractal in batik becomes the foundation of this paper to create algorithm which will produce new kind of patterns: Batik Fractal. The method used for creating the pattern is L-System and Fractal Dimension. L-System is used to create pattern, while Fractal Dimension is used as a measurement tool for Batik Fractal to compare with traditional batik. Algorithm to create Batik Fractal has been developed into a software known as jBatik. As a software, jBatik becomes a helping tool for batik makers to create new patterns. jBatik v 2.0 has been used as a tool to create new batik patterns in creative industry by involving several batik makers.
\end{abstract}

Keywords: anova test; batik fractal; jBatik; L-System; pattern.

\section{$1 \quad$ Introduction}

Batik and fractal is two different concepts. Batik is in region of art, while fractal is a mathematical concept that discuss iteration and self-similarity. However, batik and fractal joins into a concept in new kind of batik: Batik Fractal. In Batik Fractal, fractal is used to design and redesign new patterns using jBatik software.

The use of fractal in batik is not accidental, but through several research which proves that there is fractal element in batik [1]. To prove the presence of fractal characteristic in batik, we measure it using Fractal Dimension. Fractal Dimension Measurement is using Box-Counting Methods and Fourier Transformation [2].

The presence of Fractal in Batik provides inspirations to create new pattern designs based on traditional patterns and fractal formulas. jBatik helps ease the Received November $27^{\text {th }}, 2009$, Revised November $14^{\text {th }}, 2013$, Accepted for publication November $15^{\text {th }}$, 2013.

Copyright $\odot 2013$ Published by LPPM ITB,ISSN: 1978-3078, DOI: 10.5614/itbj.vad.2013.4.1.9 
creation of new patterns using fractal. jBatik Is a software that is builds using Java Programming with GUI and user friendliness for designers/artists. By using jBatik, batik design with fractal concept can be made easily. The software can even create new patterns by changing its parameters.

There is also Fractal Dimension button to quantify the fractality from Batik Fractal. This features will help to ensure the user so that the new batik will still be in range of fractal dimension of traditional batik. The latest innovation of jBatik v 2.0 is the ability to create new batik patterns in 3 dimension. This innovation will spring new possibilities in research, design and economy perspective.Furthermore, jBatik v 2.0 can also create batik patterns by filling patterns inside predefined patterns; same aspect of self-similarity process in fractal [3].

Until now, there are about 250 traditional batik that has been measured its fractal dimension. Fractal Dimension from these traditional batik is used in jBatik to check and measure the fractal dimension of new batik patterns.

The paper is organized in this order; the first part will explain methodology of measuring fractal dimension using Fourier Transformation. Second part of the methodology will explain the result of the measurement of traditional batik. In the last part, it will be presented batik designs using jBatik.

\section{$2 \quad$ Methodology}

Methodology in this paper consists of two parts. The first part is the explanation of Fractal Dimension to measure the fractality of batik, and to prove the presence of fractal in batik. The second part is the methodology to build jBatik v 2.0 .

\subsection{Fractal Dimension}

Fractal Dimension measures the fractality of an image using Box Counting Method and Fourier Transformation [1]. Dimension, in general, is defined by:

$$
D=\lim _{\epsilon \rightarrow 0} \frac{\log N(\epsilon)}{\log _{\frac{1}{\epsilon}}^{\frac{1}{4}}}
$$

If $\mathrm{Z}$ is an image with measurement $M x N$, with value for each pixel is $f(x, y)$

$$
X=[f(x, y)]
$$

Fourier Transformation of Z:

$$
F(u, v)=\frac{1}{M N} \sum_{x=0}^{M} \sum_{y=0}^{N} f(x, y) e^{-j 2 \pi(u x / M+v y / N)}
$$




$$
G(u, v)=F(u, v)-\bar{F}, \bar{F}=\frac{1}{M N} \sum_{u=0}^{M} \sum_{v=0}^{N} f(u, v)
$$

Value of each pixel is defined by the value of $G$

$$
W=\|G(u, v)\|
$$

Value for each $\mathrm{W}$, grouped in angle parameter ( $\mathrm{m}$ of different angle) and certain distance ( $\mathrm{n}$ of different distance), with reference of ordinate $\left(x_{c}, y_{c}\right)$ (see Figure $1)$ :

$$
W\left(\theta_{i}, R_{j}\right)=\left\{w_{u v} \mid \tan ^{-1} \frac{v-y_{c}}{u-x_{c}} \in \theta_{i}, \sqrt{\left(u-x_{c}\right)^{2}+\left(v-y_{c}\right)^{2}} \in R_{j}\right\}
$$

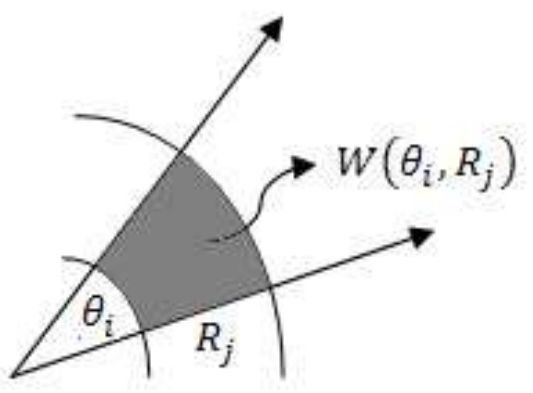

Figure 1 Coordinate dividing in angle and distance.

$$
\begin{aligned}
& D_{f}\left(\theta_{i}\right)=\alpha_{i}, \psi_{i}=-\alpha_{i} R+\delta_{i} \\
& \psi_{i}=\left\{\frac{1}{\bar{\omega}_{i}} \sum w\left(\theta_{i}, R_{j}\right) \mid j=k, \cdots, n\right\} \\
& R=\left\{R_{j} \mid j=k, \cdots, n\right\} \\
& D f=\left\{D f\left(\theta_{i}\right) \mid i=1, \cdots, m\right\} \\
& D F=\frac{1}{m} \sum_{i=1}^{m} D f\left(\theta_{i}\right)
\end{aligned}
$$

\section{2 jBatik}

jBatik is a software developed by Pixel People Project to design batik patterns using fractal formula (Figure 2). At this point, jBatik uses L-System to develop fractal for batik design.

If a batik is defined as:

Batik $\mathfrak{B}:\{$ main ornament, isen $\}$ 
Rapport $\Re$ : smallest unit of patterns, so if rapports are arranged it will create a complete pattern.

Suppose $\mathrm{G}$ is a basic geometry, $\Gamma$ is algorithm/rule to create $\mathcal{G}$ becomes ornament $\mathcal{O}$ of batik.

$$
\begin{aligned}
\Gamma(\mathcal{G}) & =\mathcal{O} \\
\Gamma: \mathcal{G} & \rightarrow \mathcal{O}
\end{aligned}
$$

And suppose $\mathcal{F}$ is an algorithm to create $\mathcal{G}$ to become isen $\mathcal{J}$ for a main ornament $\mathcal{O}$,

$$
\begin{aligned}
& \mathcal{F}(\mathcal{G}, \mathcal{O})=\mathcal{J} \\
& \mathcal{F}: \mathcal{G} \times \mathcal{O} \rightarrow \mathcal{J}
\end{aligned}
$$

Then algorithm to create a rapport can be defined as a way of placing isen on the main ornament

$$
\Re=\Re(\mathcal{O}, \mathcal{J})
$$

Thus for a batik pattern, which is the collection of several rapports with certain arrangements, can be written as:

$$
\mathfrak{B}=\mathfrak{B}(\mathfrak{R})
$$

Algorithm $\Gamma \& \mathcal{F}$ to create main ornament $\mathcal{O}$ and isen for jBatik at this moment is by using L-System. L-System is an axiomatic system which consists of variable (V), symbol (S), axiom ( $\omega)$, and rule (P). Formally, it can be written as:

$$
G=\{V, S, \omega, P\}
$$

The whole algorithm in jBatik will create a batik rapport $\Re$. We can then obtained Fractal Dimension (Eq. 11) from the rapport.

$$
D F(\Re)
$$

The value of this Fractal Dimension is then compared with the database of traditional batik's fractal dimension. For values which are outside the statistic of traditional batik's fractal dimension, the rapport will be recreated in jBatik. 


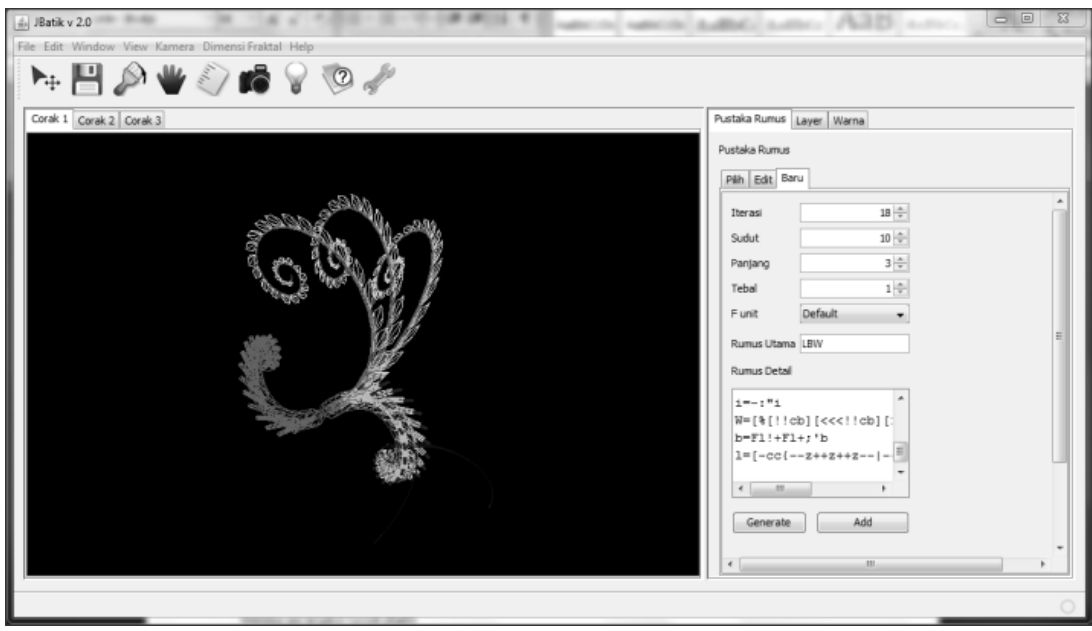

Figure 2 jBatik v 2.0 appearance. The right panel is a place to write L-System. This panel also serves as library for formulas.

\section{$3 \quad$ Analysis}

\subsection{Traditional Batik}

Fractal dimension of traditional batik is ranging around 1.5. This shows that batik has fractional dimension (not integer). Compared to Picasso painting which has fractal dimension of 3, fractal character in batik shows that batik has

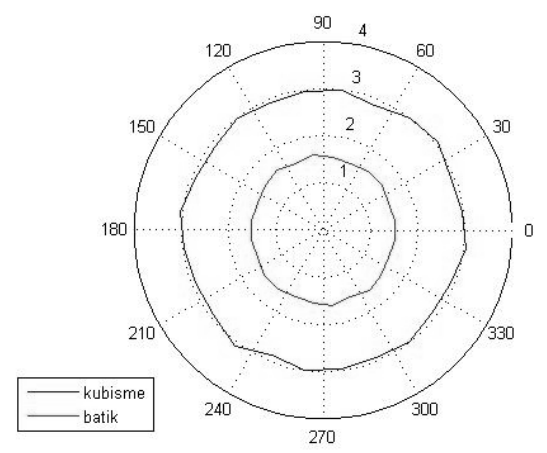

Figure 3 Fractal dimension of cubism painting by Picasso and batik. It shows that these two art forms has different characteristic. Fractal dimension of batik is half of cubism painting. 
high level of detail in various scales. Moreover, fractal character of batik spread symmetrically (except for banji pattern) and batik regions. Generally, batik has fractal characteristic. Specifically, fractal dimension of each pattern is different. Batik also has different fractal dimension according to its region where it is made.

Consistency of fractal dimension's value of batik, which is between 1 and 2, shows the sign of obeying the pakem/ rule. This obedience of pakem can be compared with cubism to draw objects (Figure 3). Besides obeying pakem, the limitation of media also contributes in maintaining the dimension in its range.

Next, we will analyse furthermore in batik pattern's aspect. Anova Testing in Fractal Dimension shows that there are four groups of patterns with different fractal dimension (Figure 4). First group with fractal dimension around 1.4 is comprised of water plant and animal pattern. Second group with fractal dimension around 1.5 is comprised of vines pattern. Third group with fractal dimension around 1.65 comprised of parang patterns, geometric pattern and flowers. Fourth group with fractal dimension around 1.8 comprised of banji pattern.

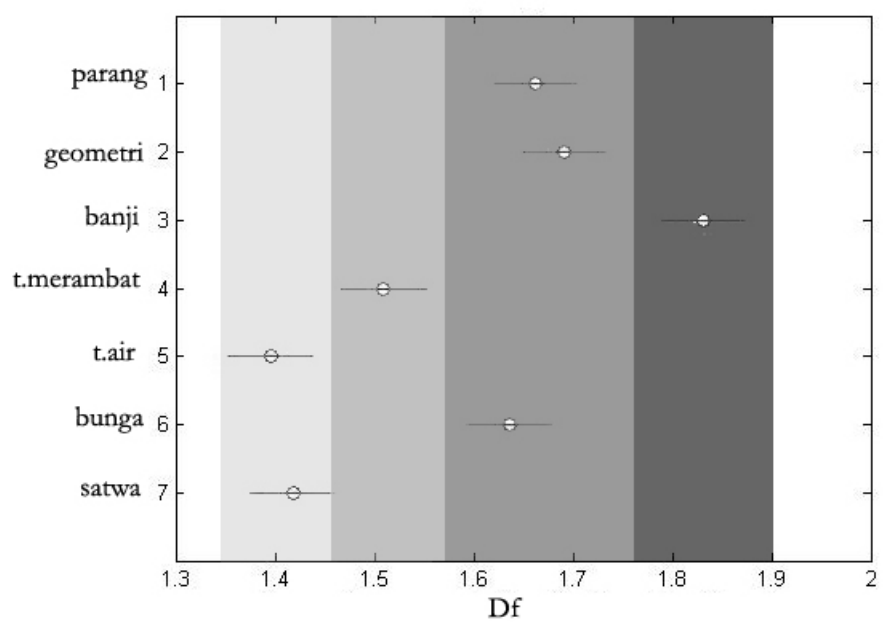

Figure 4 Anova Test shows that there are four groups according to its fractal dimensions, with confidence level $95 \%$.

In region aspect, batik also shows the consistency of fractal characteristic, which is having a fractal dimension between 1 and 2 (Figure 5). There are five groups of batik according to its region. Batik Jogja and batik Solo is an intersection between batik Madura and batik Garut. Batik Lasem is in one group 
with Batik Tasik, and Batik Cirebon is in its own group, without any similarity with other region.

The presence of fractal in batik raises further question: why batik has fractal characteristic? To answer this question, one must understand the character of fractal itself. The presence of fractal means that there is self-similarity and selfaffine in the patterns. These characteristic in batik means that exists geometric detailing in various smaller scales. In batik, isen process contributes significantly to detailing in smaller scales.Because the isen and main pattern does not have to be geometrically similar, then the process is closely related to self-affine than to self similarity.

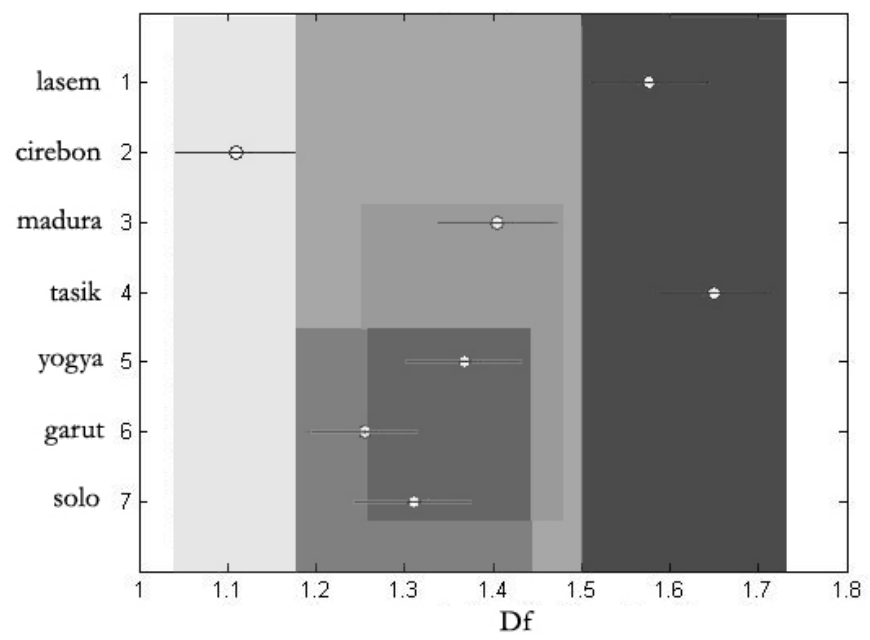

Figure 5 Anova Test shows the grouping of fractal dimension according to its region. Batik Cirebon has fractal dimension which is different with other regions.

\section{$4 \quad$ Batik Fractal in jBatik}

In this part, fractal dimension of Batik Fractal will be compared with traditional batik pattern. Figure 6 shows the distribution of Fractal Dimension (Df) of Batik Fractal among traditional batik.So far, Batik Fractal's dimension is consistent between 1 and 2 ("kawung asli" pattern, drawn with L-System). In several pattern of Batik Fractal, the dimension ranges between 2 and 3 (3 dimensional "kawung" patterns drawn with L-System ). Several variations of these two patterns ("kawung asli" and "kawung") will alter its dimension (Figure 7). These variations can be done by changing its parameter such as: angle, basic formula and iterations. 


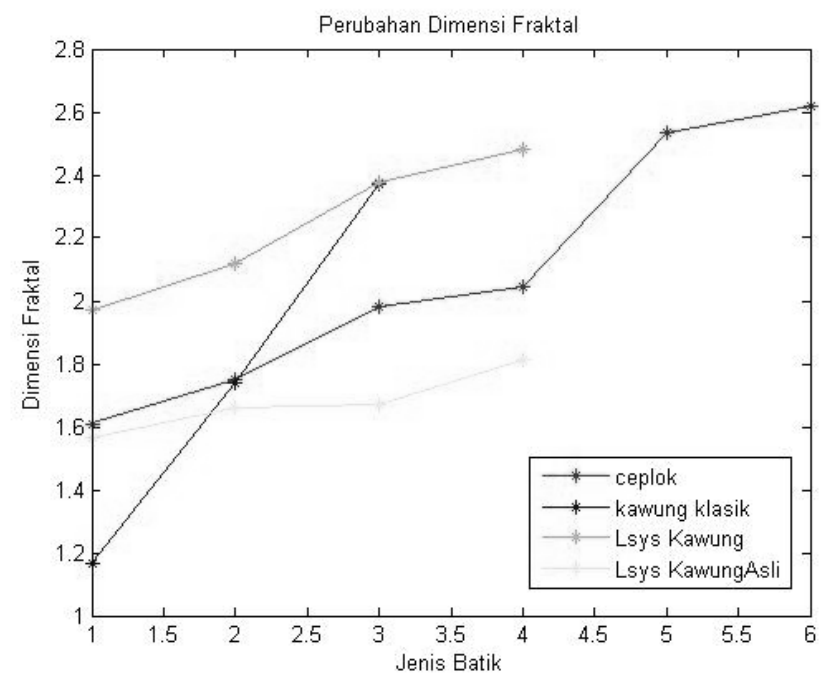

Figure 6 Differentiation of fractal dimension scattering, from classic to LSystem. Kawung L-System is in region of classic kawung and ceplok. Ceplok pattern is in region of classic kawung.

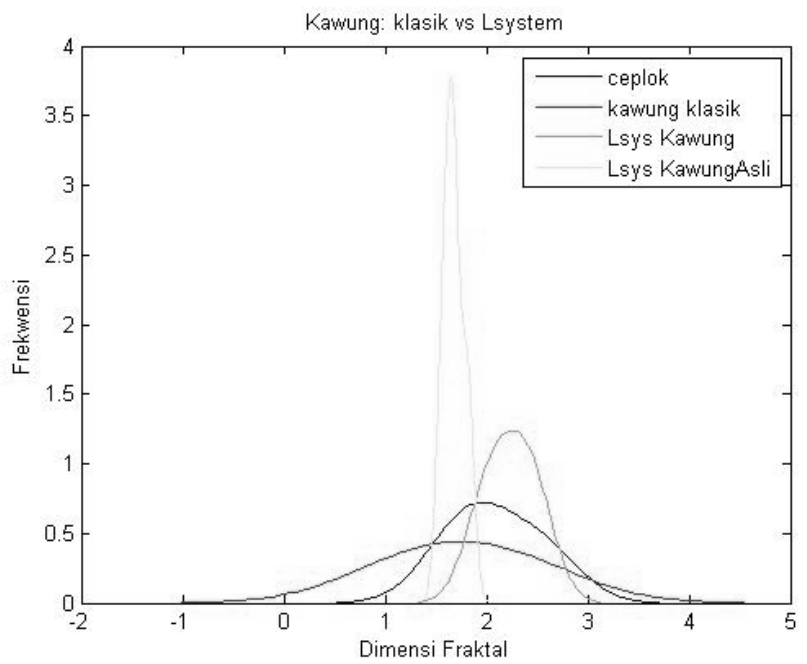

Figure 7 The changing of fractal dimension in several kinds of kawung patterns, sequenced from smallest to biggest one. DF pattern of classic kawug spreads from 1.2 to 2.4. DF LSystem kawung is divided into two parts, one part is above the ceplok pattern, while other group is below the ceplok pattern. 


\section{Discussion, Suggestion and Conclusion}

Batik has fractal dimension between 1 and 2. Fractal dimension has fractional value which gives inspiration to develop batik using fractal. By developing jBatik Software, fractal has been deliberately and continuously used to create Batik Fractal.

jBatik is a software with Java Language Programming to generate Batik Fractal. To accommodate distinct characters of batik, jBatik has developed parameters to suit those characters such as pattern, isen, colour, and fractal dimension.

Fractal dimension to measure fractality of batik can be known by using Box Counting Method and Fourier Transformation. Fractal dimension is implanted in jBatik as a tool to control the fractality of batik so as it would not stray from traditional batik.

Innovation of $\mathrm{jBatik}$ (at this moment is $\mathrm{v} 2.0$ ) covers the area to create batik 3D, importing 2 dimensional and 3 dimensional objects as a unit of fractal pattern. It also covers exporting 2 dimensional file, and also 3 dimensional file for further uses such as RPM. Layouting the pattern in more designer-like GUI interface allows designers/ artists to create batik pattern. There is also importing pattern inside pattern to create self-similarity easily.

In batik, isen process contributes significant aspect in creating detailing in smaller scales. Because the main pattern and isen does not have to be geometrically similar, the process is closely related to self-affine than to selfsimilarity.

The result of Anova Test shows that batik patterns has similarity in fractal dimension, both in region aspect and in pattern. The grouping of region according to its fractal dimension resulted in 5 groups. Whereas the grouping in pattern aspects resulted in 4 groups.

To this date, jBatik v 1.0 has been radically developed to jBatik v 2.0. This development is involving participations of traditional batik makers.

The development of jBatik $v 2.0$ is far from perfect from being the ultimate batik pattern generator. There are many research yet to be implemented in creating algorithm for defining rules of batik in jBatik.

\section{Acknowledgement}

Author gratefully acknowledges to colleagues of Pixel People Project for tirelessly developing jBatik v 2.0 so that it can be used as an object for this 
paper. Author also gratefully acknowledges USAID-Senada for grant in developing jBatik v 2.0

\section{References}

[1] Hariadi, Y., Lukman, M. \& Haldani, A. 2007. Batik Fractal: From Traditional Art to Modern Complexity, Proceeding Generative Art X Milan Italia.

[2] Heurteaux, Y. \& Jaffard, S. 2007. Multifractal Analysis of Images: New Connexions between Analysis and Geometry, J. Byrnes (ed.), Imaging for Detection and Identification, pp. 169-194, Springer.

[3] Aouidi, J. \& Slimane, M.B. 2002. Multi-Fractal Formalism for QuasiSelf-Similar Functions, Journal of Statistical Physics, 108(3/4). 